\title{
Evaluation of kalrn transcript levels in zebrafish seizure model
}

\author{
Marcela M. Simões (IC), Patrícia G. Barbalho (PQ), Cláudia V. Maurer-Morelli (PQ).
}

\author{
Abstract \\ Temporal transcript profile of kalrn gene in zebrafish larvae brain after pentylenotetrazole (PTZ)-induced \\ seizure.
}

Key words: zebrafish, kalrn, seizure.

\section{Introduction}

Kalirin (kalrn) regulates neuronal shape and spine growth playing an important role in plasticity [1]. Besides, it has been implicated in some neurological diseases [2]. Today, there is no study associating the kalrn gene and seizures. We have been using the zebrafish seizure model for genetic investigations because its advantages in genetics studies. The main aim of this study was to investigate the kalrn mRNA levels in the zebrafish pentylenetetrazole (PTZ)-seizure model.

\section{Results and Discussion}

Our results showed no statistical difference in the kalrn mRNA levels between seizure group (SG) and control group (CG) after 24 and 48 hours of PTZ-induced seizure (images 1 and 2). The mean \pm SEM of karln mRNA comparing SG and CG groups were the following: CG24h $0.85 \pm 0.14$; SG24h $0.92 \pm 0.1 \quad(p=0.5)$ and CG48h 1.00 \pm 0.03 ; SG48h $0.93 \pm 0.03(p=0.1)$.

A

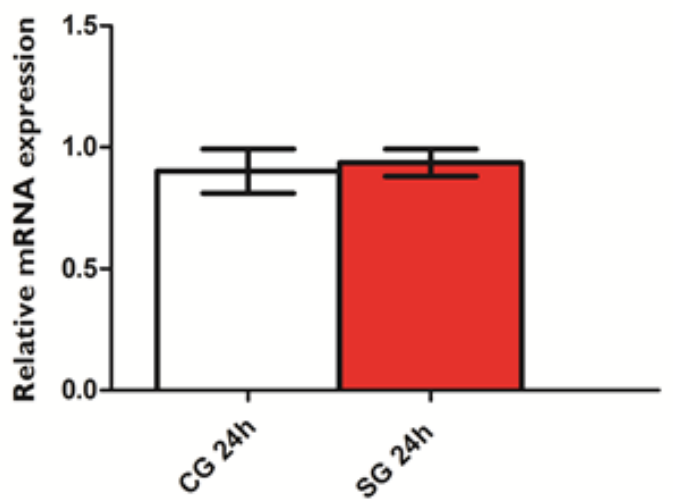

Image 1. Relative quantification of kalrn mRNA in zebrafish larvae brain after 24 hours after pentylenetetrazole-evoked seizure.
B

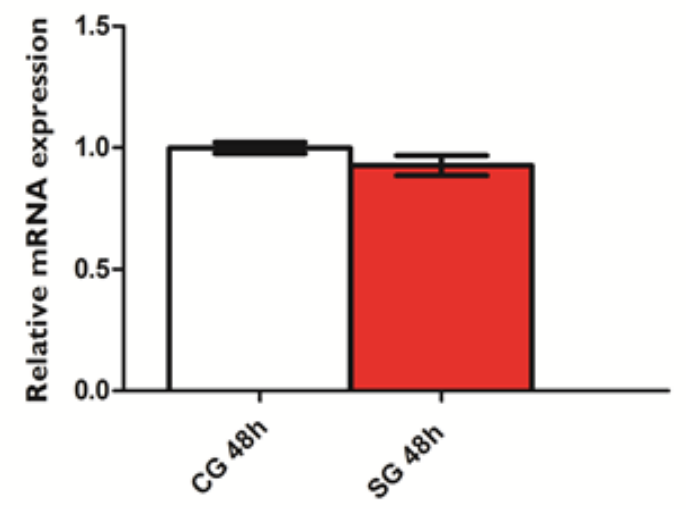

Image 2. Relative quantification of kalrn mRNA in zebrafish larvae brain after 48 hours after pentylenetetrazole-evoked seizure.

The kalrn gene has been associated with human neurological disorders, such as schizophrenia, Alzheimer disease, Huntington's disease and ischemic stroke[2]; nevertheless, there is no information about this gene and epilepsy. Although no differences were found in karln transcripts at $24 \mathrm{~h}$ and $48 \mathrm{~h}$ after PTZ, we cannot conclude that karln does not play a role after seizures.

\section{Conclusions}

By investigating other time points of karln mRNA expression we may shed some light into the role of this gene and its relation with the mechanisms underlying plasticity in epilepsy.

\section{Acknowledgement}

I would like to greatly thank the patience and help of my supervisor Claudia Morelli, and also Patricia Barbalho and all members of zebrafish lab. Suported by: PIBIC-CNPq and BRAINNCEPID/FAPESP, BRAZIL

\footnotetext{
${ }^{1} \mathrm{Ma} \mathrm{XM}$ et al. Kalirin-7 is required for synaptic structure and function. J Neurosci 2008, 28, 12368-12382.

${ }^{2}$ Penzes P, Remmers C. Kalirin Signaling: Implications for Synaptic Pathology. Mol Neurobiol 2012, 45(1), 109-118.
} 